\title{
The role of indoleamine 2,3-dioxygenase in LP-BM5 murine retroviral disease progression
}

\author{
Megan A O'Connor ${ }^{1}$ and William R Green ${ }^{1,2^{*}}$
}

\begin{abstract}
Background: Indoleamine 2,3-dioxygenase (IDO) is an immunomodulatory intracellular enzyme involved in tryptophan degradation. IDO is induced during cancer and microbial infections by cytokines, ligation of costimulatory molecules and/or activation of pattern recognition receptors, ultimately leading to modulation of the immune response. LP-BM5 murine retroviral infection induces murine AIDS (MAIDS), which is characterized by profound and broad immunosuppression of T- and B-cell responses. Our lab has previously described multiple mechanisms regulating the development of immunodeficiency of LP-BM5-induced disease, including Programmed Death 1 (PD-1), IL-10, and T-regulatory (Treg) cells. Immunosuppressive roles of IDO have been demonstrated in other retroviral models, suggesting a possible role for IDO during LP-BM5-induced retroviral disease progression and/or development of viral load.

Methods: Mice deficient in IDO (B6.IDO-/-) and wildtype C57BL/6 (B6) mice were infected with LP-BM5 murine retrovirus. MAIDS and LP-BM5 viral load were assessed at termination.

Results: As expected, IDO was un-inducible in B6.IDO-/- during LP-BM5 infection. B6.IDO-/- mice infected with LP-BM5 retrovirus succumbed to MAIDS as indicated by splenomegaly, serum hyper IgG2a and IgM, decreased responsiveness to $\mathrm{B}$ - and T-cell mitogens, conversion of a proportion of $\mathrm{CD}^{+} \mathrm{T}$ cells from Thy $1.2^{+}$to Thy $1.2^{-}$, and increased percentages of $\mathrm{CD} 11 \mathrm{~b}^{+} \mathrm{Gr}-1^{+}$cells. LP-BM5 infected B6.IDO-/- mice also demonstrated the development of roughly equivalent disease kinetics as compared to infected B6 mice. Splenic viral loads of B6 and B6.IDO-/mice were also equivalent after infection as measured by LP-BM5-specific Def Gag and Eco Gag viral mRNA, determined by qRT-PCR.
\end{abstract}

Conclusions: Collectively, these results demonstrate IDO neither plays an essential role, nor is required, in LP-BM5 -induced disease progression or LP-BM5 viral load.

Keywords: LP-BM5 murine retrovirus, Indoleamine 2,3-dioxygenase, Murine AIDS

\section{Background}

Indoleamine 2,3-dioxygenase is an immunomodulatory intracellular enzyme involved in the first, rate-limiting step of tryptophan catabolism [1]. IDO expression is induced by a variety of immune responses, including upregulation of type I $(\alpha, \beta)$ and type II $(\gamma)$ interferons (IFNs), and following toll-like receptor (TLR) ligation [2-5]. IDO can also be activated upon ligation of B7 costimulatory molecules (CD80 and CD86) on dendritic cells (DCs) by CD28 or CTLA-4 on T-cells [1,3,5-7].

\footnotetext{
* Correspondence: William.R.Green@dartmouth.edu
'Department of Microbiology and Immunology, Geisel School of Medicine at

*Correspondence: William.R.Green@dartmouth.edu
'Department of Microbiology and Immunology, Geisel School of Medicine at Dartmouth, Lebanon, New Hampshire 03756, USA

${ }^{2}$ Norris Cotton Cancer Center, Geisel School of Medicine at Dartmouth, Lebanon, New Hampshire 03756, USA
}

Upon induction, high levels of IDO expression are primarily found in a specialized subset of murine plasmacytoid dendritic cells (pDCs) expressing $\mathrm{CD} 8 \alpha, \mathrm{CD} 19$, and B220, but can also be found in other cell types including macrophages, epithelial cells, and some fibroblast-like cells [7-10].

IDO expression induces tryptophan catabolism, resulting in reduced tryptophan levels and increased levels of toxic downstream metabolites (kynurenine, quinolic acid, and picolinic acid), all of which can contribute to T-cell suppression $[8,11,12]$. T-helper 1 (Th1) cell clones are more sensitive to changes in tryptophan levels than T-helper 2 (Th2) cell clones, resulting in increased Th1directed immunosuppression [7]. Furthermore, IDO 
induction is associated with altering the balance of $\mathrm{T}$ cell subsets, increasing the proportion of T-regulatory (Treg) cells and decreasing T-helper 17 (Th17) cells $[2,13,14]$. Overall, IDO induction causes immunosuppression by dampening Th1 and Th17 cellular responses and enhancing regulatory $\mathrm{T}$-cell responses.

The immunosuppressive role of IDO in modulating immune responses to cancer is well recognized, but the role of IDO during viral infections is less well established [15]. In microbial infections, IDO can act as either an immune suppressor or as an anti-microbial agent, depending on the nature of the infection. IDO-linked immunosuppression occurs during in vitro Puumala hantavirus infection [16] and in vivo during Plasmodium species [17] and Leishmania major infections [18]. In contrast, IDO induction acts in vitro and/or in vivo against bacteria, such as Chlamydiae, Streptococci and Staphylococci species [19-21]; parasites, for example Toxoplasma gondii [21]; and viruses, including herpes simplex-2 virus (HSV-2), cytomegalovirus (CMV), hepatitis $B$ virus (HBV), and vaccinia virus infections [22-25]. Increased IDO expression occurs during human immunodeficiency virus (HIV) [26-29], simian immunodeficiency virus (SIV) $[13,30]$ and murine LP-BM5 immunodeficiency-causing retroviral infections [31], yet a comprehensive understanding of the effect of IDO on these retroviral infections has not been provided. Increased IDO mRNA levels in HIV patients have been correlated with increased viral loads, and IDO expression decreases upon antiretroviral therapy (ART), suggesting a direct correlation between IDO and HIV virus propagation [27]. Additional studies in HIV/AIDS systems have further suggested that IDO may play an active immunosuppressive role $[7,32,33]$.

LP-BM5 retroviral infection causes a profound and broad immunodeficiency in susceptible mouse strains such as C57BL/6 (B6); this disease is known as murine AIDS (MAIDS). MAIDS is characterized by early polyclonal T- and B-cell activation, splenomegaly, lymphadenopathy, hypergammaglobulinemia, and a subsequent progressive immunodeficiency of T- and B-cell responses [34-37]. As with AIDS patients, this immunodeficiency leads to an increased incidence of B-cell lineage lymphomas and susceptibility to a variety of opportunistic pathogens [34,38]. In resistant BALB/C mice, we have demonstrated a key protective role of Gag-specific $\mathrm{CD} 8^{+}$ cytotoxic T-lymphocytes (CTLs), which are critical for controlling LP-BM5 infection and protecting against retroviral pathogenesis [38-42]. We have not been able to detect such robustly protective CTLs in the susceptible B6 model, strongly suggesting that this CTL deficit is critical to the disease induction.

In the LP-BM5 retroviral system immunosuppressive mechanisms have been identified which modulate disease progression. The kinetics and degree of MAIDS pathogenesis are increased after LP-BM5 infection of B6 mice deficient for the Programmed Death 1 (PD-1) gene, but disease can be decreased if instead the interruption of PD-1 signaling is confined to the CD8 compartment $[43,44]$. Elevated splenic anti-inflammatory IL-10 mRNA levels are found in wildtype (w.t.) B6 mice following LPBM5 infection [45]. Mice deficient of the IL-10 gene are more susceptible to MAIDS than w.t. B6 mice, presumably because, as we have shown for PD-1, IL-10 may well act to control pathogenic $\mathrm{CD} 4^{+} \mathrm{T}$-cells that drive LP-BM5-induced disease [43]. Our lab has examined the role of $\mathrm{CD}_{4}^{+}$Tregs, and demonstrated that Forkhead box P3 (FoxP3) ${ }^{+} \mathrm{CD} 4^{+}$T-cells increase during the first 2 weeks of infection and plateau at about 18 days post infection (dpi) [44]. Upon initial depletion of natural Tregs, via adoptive transfer of FoxP3 ${ }^{-} \mathrm{CD} 4^{+} \mathrm{T}$ cells into B6.TCR $\alpha \mathrm{R}-/$ - mice, which lack $\mathrm{T}$ cells, there was no substantial change in disease severity [44]. However, MAIDS pathogenesis can be reduced upon depletion of FoxP3 ${ }^{+} \mathrm{CD} 4^{+}$Treg cells, in combination with elimination of PD-1 expression on $\mathrm{CD}^{+} \mathrm{T}$-cells [44]. This reduction in the degree of MAIDS seen was apparently due to the observed expansion of a protective $\mathrm{CD} 8^{+}$CTL population, which had previously been suppressed by FoxP3 ${ }^{+}$ Tregs and/or PD-1 following the infection of intact, unmanipulated B6 mice. These studies further suggest an important role of immunosuppressive mechanisms in either the promotion of, or protection from, LP-BM5-induced pathogenesis.

We have also identified an immunosuppressive monocytic myeloid-derived suppressor cell (MDSC) po-

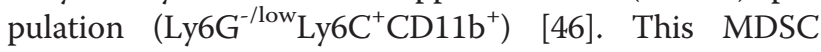
population increases during LP-BM5 infection and is capable of suppressing both $\mathrm{T}$ - and B-cell responses, in significant part via an inducible nitric oxide synthase (iNOS)-dependent manner [46]. It is possible that the immunosuppressive mechanism(s) of MDSCs in B6 mice could be regulated during LP-BM5 infection, due to the known cross-talk between the iNOS pathway and tryptophan catabolism in other systems [9,11]. Downstream metabolites of tryptophan catabolism can alter iNOS expression; 3-hydroxyanthranilic acid (3HAA) is able to inhibit the activity and expression of iNOS; while picolinic acid is able to induce iNOS in conjunction with IFN $\gamma$ activation [47,48]. In short, it is becoming appreciated that immunoregulatory mechanisms affect LP-BM5-induced disease progression, suggesting that immunosuppressive roles of IDO could also be playing an important role in MAIDS pathogenesis.

We assessed the role of IDO inhibition on MAIDS progression and LP-BM5 viral load. Compared to other immunodeficiency-causing retroviral infections in primate species, the LP-BM5 murine retroviral system afforded the opportunity for a more comprehensive assessment of 
IDO, including the use of B6.IDO-/- mice. Our studies suggest a non-essential role for IDO in LP-BM5-induced disease, with little, if any, effect on retroviral load.

\section{Results and Discussion}

IDO is undetectable in B6.IDO-/- mice following LP-BM5 infection

The genotype of B6.IDO-/- mice was evaluated, to confirm the absence of the normal w.t. IDO gene. PCRamplified products of genomic DNA were run on agarose gels to identify the presence of either w.t. or knockout IDO DNA. The wildtype IDO DNA signature (427 bp) was observed only in B6 mice, and the characteristic knockout IDO DNA fragment (280 bp) was found only in B6.IDO-/- mice (Figure 1A). Next, the possible induction of IDO during LP-BM5 infection was determined. B6 and B6.IDO-/- mice were infected in parallel with LP-BM5, and splenic mRNA was assessed at 8 weeks post infection (wpi) for the presence of IDO message using qRT-PCR (Figure 1B). As expected, IDO
mRNA was undetectable in all uninfected and infected B6.IDO-/- mice. In contrast, minimal levels of IDO mRNA were detected in uninfected w.t. B6 mice and significantly increased by 8 wpi (Figure 1B). Plasmacytoid dendritic cells ( $\mathrm{pDCS}$ ) have been shown to be major producers of IDO [8], but in our preliminary experiments, no overall obvious difference was seen in the percentage of the pDC cell population at 8 wpi in B6 and B6.IDO-/- mice (data not shown). The induction of IDO in B6 mice after LP-BM5 infection is consistent with a possible role for IDO in LP-BM5-induced pathogenesis and/or viral load.

\section{B6.IDO-/- mice succumb to MAIDS after LP-BM5 infection}

To assess the role of IDO in modulating LP-BM5-induced disease, B6 and B6.IDO-/- mice were infected, in parallel, with LP-BM5 and evaluated at 8 wpi for standard MAIDS disease read-outs. Severe splenomegaly occurred in infected B6 and B6.IDO-/- mice at 8 wpi (Figure 2A),

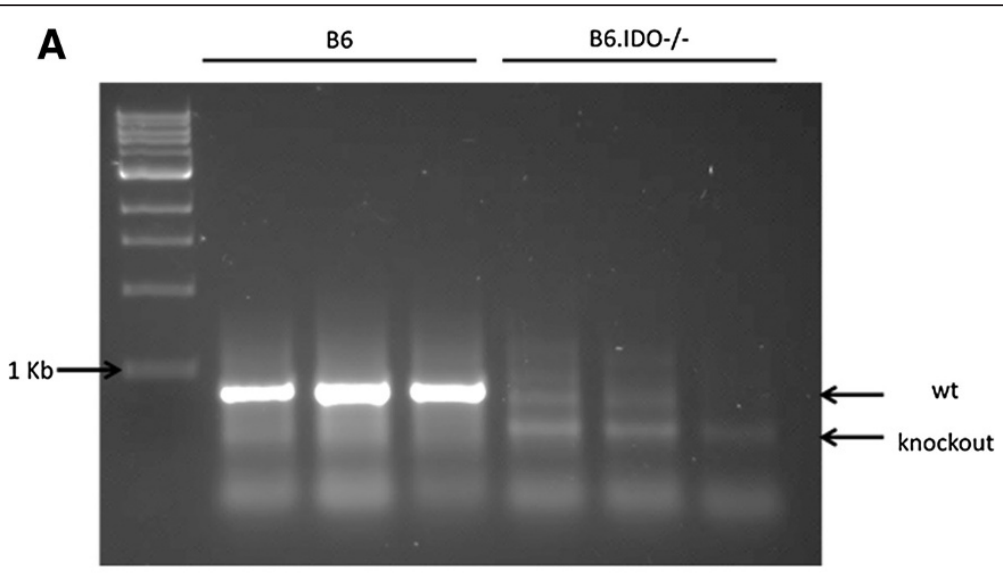

B

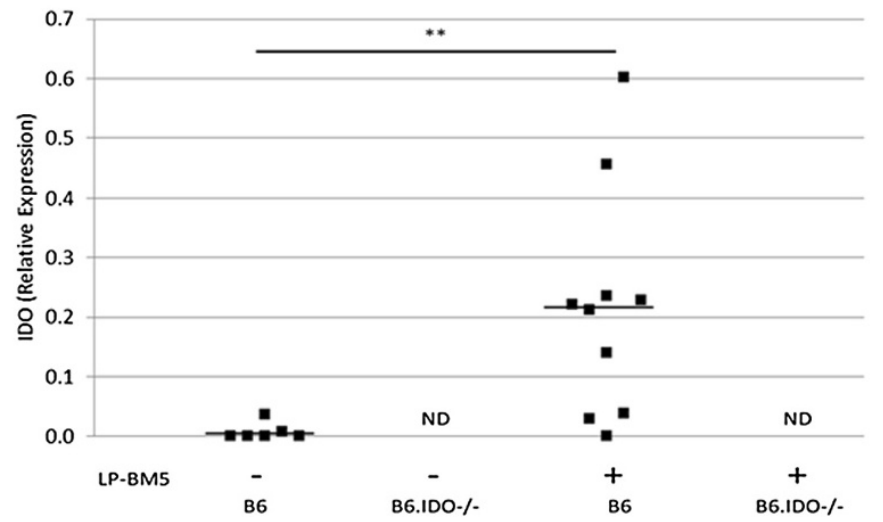

Figure 1 IDO is Undetectable in B6.IDO-/- Mice Following LP-BM5 Infection. (A) Genomic DNA was isolated from B6 and B6.IDO-/- mice, and the IDO genotypes were determined using PCR. Expected IDO band lengths: w.t. IDO (427 bp) and knockout IDO (280 bp). (B) Relative expression of IDO splenic mRNA by qRT-PCR, as compared to $\beta$-actin. Lines represent the mean, $n=10$, from three independent experiments. IDO mRNA was not detected (ND) in uninfected or infected B6.IDO-/- mice. ${ }^{* *} \mathrm{p}<0.01$, in comparison to uninfected controls, as determined using one-way ANOVA. 

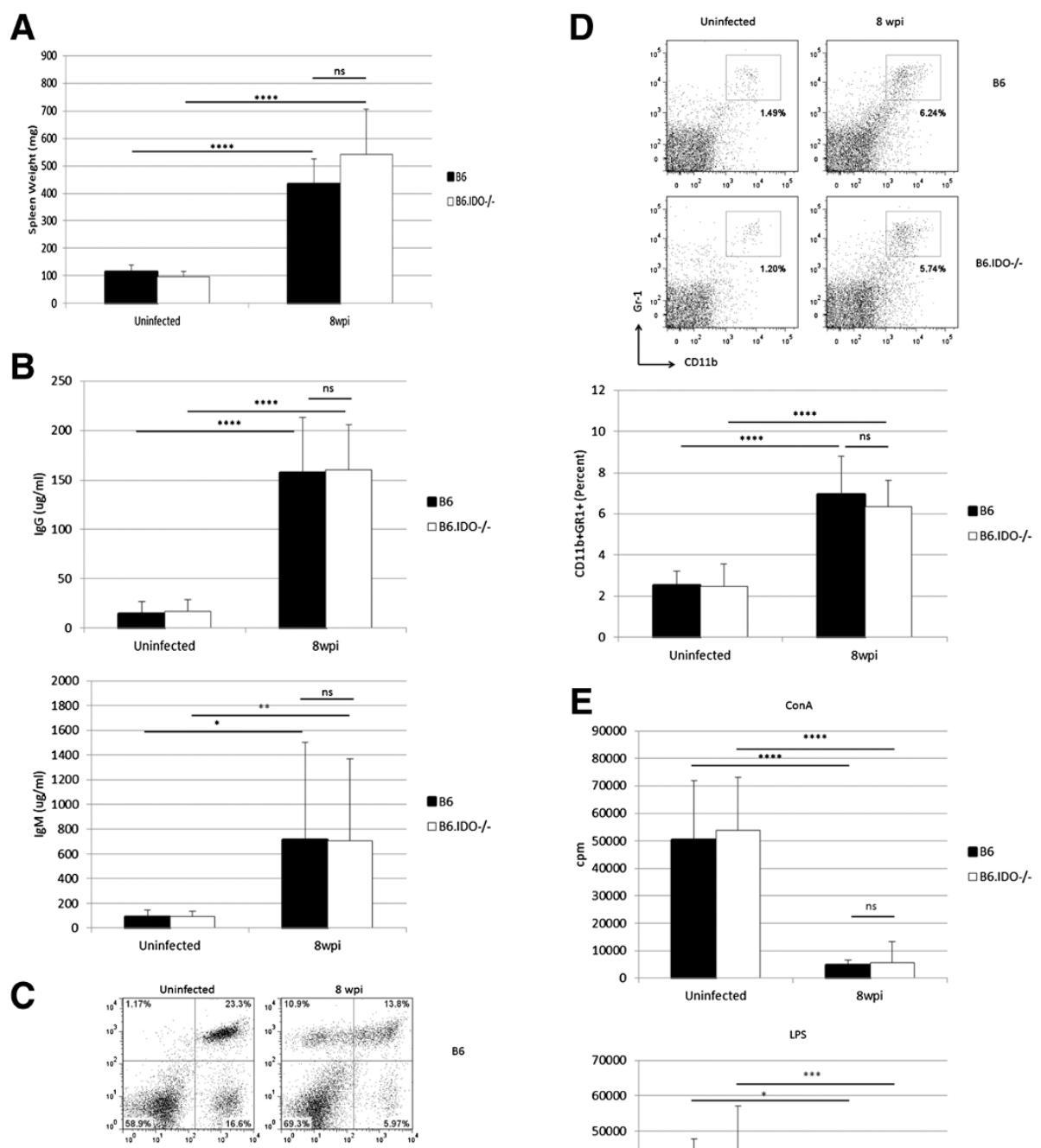

B6
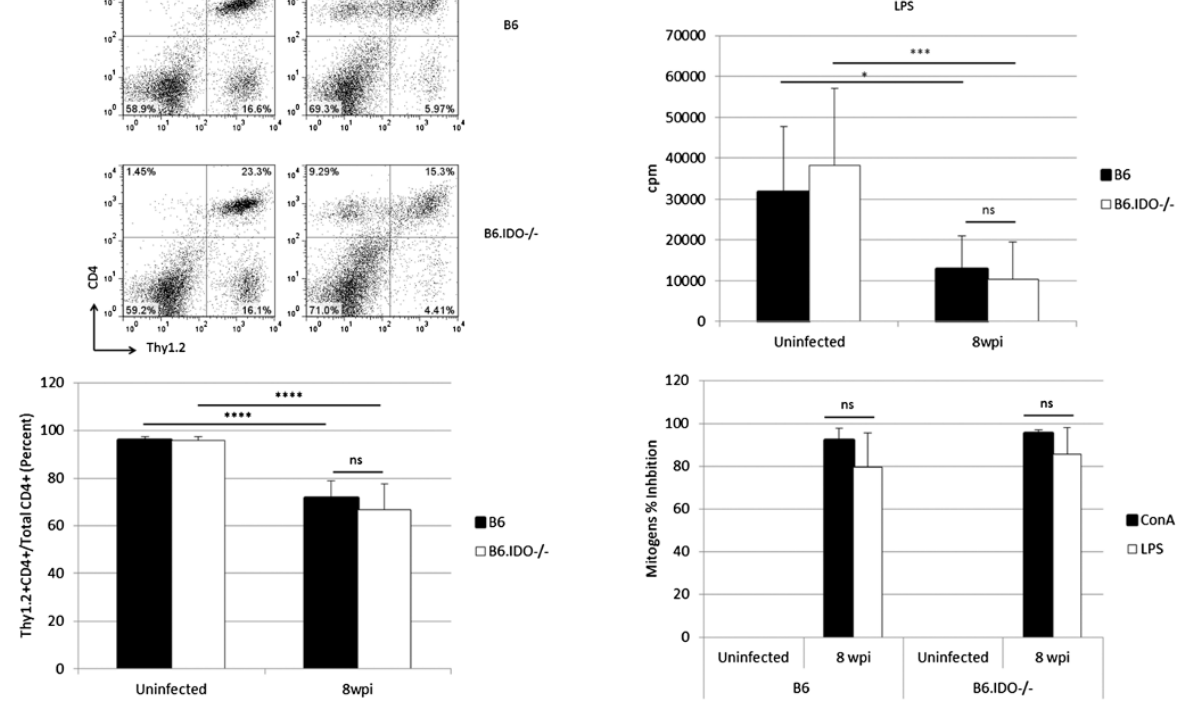

Figure 2 B6.IDO-/- Mice Succumb to MAIDS After LP-BM5 Infection. B6 and B6.IDO-/- mice were infected in parallel, with $5 \times 10^{4}$ pfu of LP-BM5, and MAIDS parameters were assessed at 8 wpi. (A) Spleen weights. (B) Serum IgG2a and IgM as assessed by ELISA. (C) Emergence of Thy $1.2^{-} \mathrm{CD} 4^{+}$T-cells as assessed by flow cytometry. Percent of Thy $1.2^{+} \mathrm{CD} 4^{+}$of the total $\mathrm{CD} 4^{+}$cell population, calculated from the flow plots of all the experiments. (D) Increase in the CD11 b+GR-1 $1^{+}$cell population, assessed by flow cytometry. Percent of CD $11 b^{+} G R-1^{+}$of total splenocyte population, calculated from the flow plots of all the experiments. (E) Thymidine incorporation after stimulation with ConA and LPS, counts per minute (cpm). Mitogen percent inhibition of the uninfected control to ConA and LPS responses is shown. All histograms represent the mean of samples from four independent experiments, with indicated standard deviations. ${ }^{*} p<0.05 ;{ }^{* *} p<0.01,{ }^{* *} p<0.001$; ${ }^{* * *} p<0.0001$, in comparison to uninfected controls, as determined using one-way ANOVA. 
the standard time-point for assessment of robust disease $[43,49]$. As another parameter of the activational aspects of LP-BM5-induced pathogenesis, sera were assessed for IgG2a and IgM levels by ELISA (Figure 2B). MAIDSassociated hypergammaglobulinemia was observed at 8 wpi in both B6 and B6.IDO-/- mice, for IgG2a and IgM. During infection of B6 mice with LP-BM5, there is also the emergence of splenic $\mathrm{CD}^{+}{ }^{+} \mathrm{T}$-cells that do not express the characteristic Thy1.2 marker [34,50,51]. A significant decrease in the percentage of Thy $1.2^{+} \mathrm{CD} 4^{+}$cells of the total $\mathrm{CD} 4^{+}$population was observed in w.t. B6, as expected, but also in B6.IDO-/- mice by $8 \mathrm{wpi}$ (Figure 2C). As another indicator of LP-BM5 pathogenesis during $\mathrm{B} 6$ infection, our lab has identified an increase in a $\mathrm{CD} 11 \mathrm{~b}^{+} \mathrm{Gr}-1^{+}$cell population, as early as 5 wpi [46]. Here, we further demonstrate B6.IDO-/mice also have a significant increase in the $\mathrm{CD} 11 \mathrm{~b}^{+} \mathrm{Gr}-1^{+}$ cell population at 8 wpi (Figure 2D). Because it is a monocytic MDSC subpopulation ( $\mathrm{Ly}_{6 \mathrm{G}}{ }^{-/ \mathrm{low}} \mathrm{Ly}_{6 \mathrm{C}} \mathrm{CD}^{+} 1 \mathrm{~b}^{+}$) from LP-BM5 infected B6 mice that is capable of suppressing $\mathrm{T}$ - and $\mathrm{B}$-cell responses, in part via an iNOSdependent manner [46], we also demonstrated here that LP-BM5 infection-dependent increases in the Ly6G ${ }^{-/ l o w} \mathrm{Ly}_{6 \mathrm{C}} \mathrm{CD}^{+} 11 \mathrm{~b}^{+}$population are readily detectible in B6.IDO-/-, as well as B6, mice (data not shown). Furthermore, in preliminary experiments, Ly6 $6 \mathrm{G}^{-} \mathrm{CD} 11 \mathrm{~b}^{+}$ MDSCs derived from infected B6.IDO-/- mice have roughly similar suppressive capabilities against the response to polyclonal LPS stimulation in comparison to MDSCs derived from infected B6 mice (data not shown).

Lastly, immunosuppression was measured, as demonstrated by the standard MAIDS read-out of T- and Bcell responsiveness to polyclonal mitogen activation [51]. Isolated splenocytes from uninfected and infected mice were stimulated with ConA or LPS, and proliferation was assessed 72 hours post stimulation. At 8 wpi, B6 mice have a significantly decreased responsiveness to ConA and LPS (Figure 2E). B6.IDO-/- mice also exhibit significant and comparable levels of immunosuppression in response to these $\mathrm{T}$ - and $\mathrm{B}$-cell mitogens, as compared to uninfected controls (Figure 2E).

Based on the collective assessment of all MAIDS parameters, B6.IDO-/- mice are susceptible to MAIDS and experience immunodeficiency with similar severity to $\mathrm{B} 6$ mice.

\section{IDO is not required for MAIDS at different timepoints or doses of LP-BM5}

Although IDO thus appears to have little overall effect on MAIDS at $8 \mathrm{wpi}$, the kinetics of disease progression may differ for B6 and B6.IDO-/- mice. Timepoints earlier than the standard 8 wpi endpoint determination were therefore assessed. In these experiments, to better compare overall disease levels, mice were first evaluated for standard MAIDS parameters, including splenomegaly, hypergammaglobulinemia, responsiveness to $\mathrm{T}$ - and B-cell mitogens, and emergence of Thy $1.2^{-} \mathrm{CD} 4^{+}$ T-cells, as described in Figure 2. Then disease index, as previously described, was calculated, ranging from 0 (no disease) to 5 (severe disease) [41]. At the standard dose of $5 \times 10^{4}$ pfu of LP-BM5, disease kinetics between B6 and B6.IDO-/- mice were generally similar, with little disease at 3 wpi and a gradual increase in disease over the course of infection as assessed at 5 and 8 wpi (Figure 3A). No significant difference in the extent of disease was demonstrated between the infected groups at any of the time points.

It was next determined if B6 and B6.IDO-/- mice have different susceptibilities to disease after infection by lower viral doses. B6 and B6.IDO-/- mice were
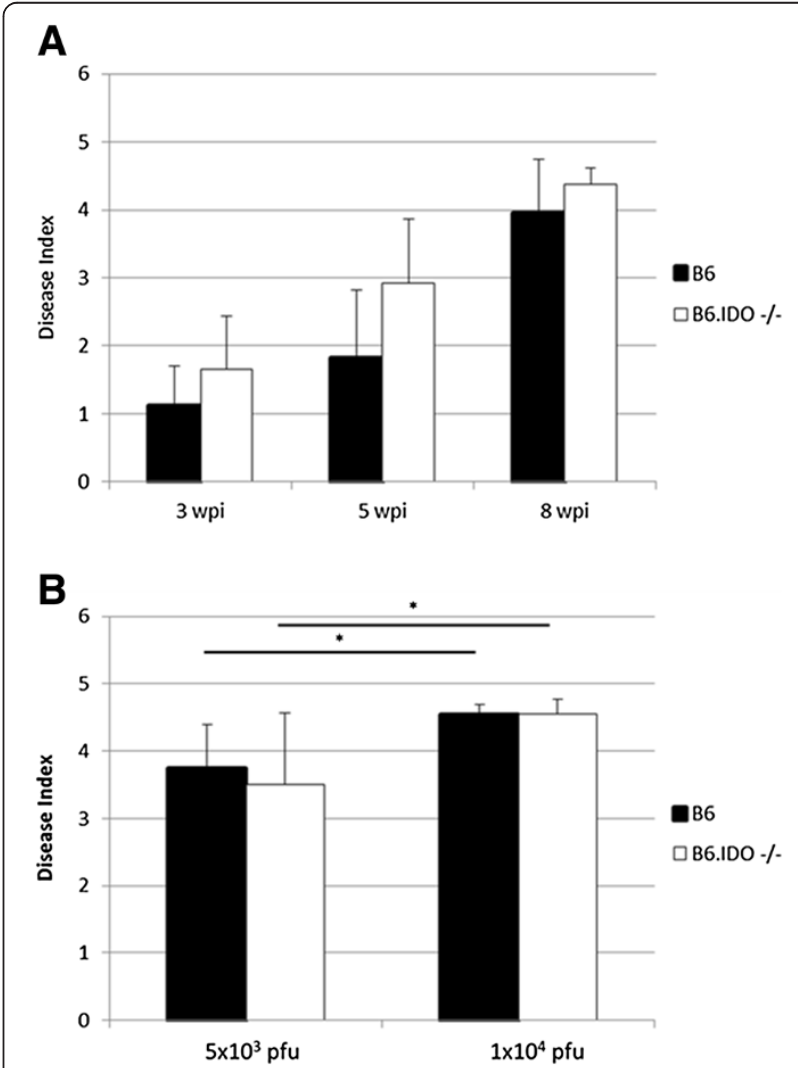

Figure 3 IDO is Not Required for MAIDS at Different Timepoints or Doses of LP-BM5. LP-BM5-induced disease kinetics were assessed in $\mathrm{B} 6$ and B6.IDO-/- mice. (A) B6 and B6.IDO-/mice were infected in parallel, with $5 \times 10^{4}$ pfu of LP-BM5 virus. MAIDS parameters were assessed at 3,5, and 8 wpi and disease index was determined. (B) B6 and B6.IDO-/- mice were infected with LP-BM5 at a dose of $5 \times 10^{3}$ or $1 \times 10^{4} \mathrm{pfu}$. Disease parameters were assessed at 8 wpi and disease index was calculated. Histograms represent the mean, $(n=3)$, with indicated standard deviations. The patterns of the presented results are representative of two additional experiments, which provided a similar pattern of results. ${ }^{*} p<0.05$, in comparison to $5 \times 10^{3} \mathrm{pfu}$, as determined using one-way ANOVA. 
infected in parallel with either $5 \times 10^{3}$ or $1 \times 10^{4}$ pfu of LP-BM5, and disease index was assessed at 8 wpi (Figure 3B). Infected B6 and B6.IDO-/- mice succumbed to substantial, and roughly equivalent, MAIDS at these two lower viral inoculums, with no observed significant differences in the quantification of disease. Thus, B6.IDO-/- mice are generally as susceptible to MAIDS at multiple viral doses as compared to prototypic susceptible B6 mice.

\section{Infected B6.IDO-/- mice do not display an altered LP-BM5 viral load}

While no apparent differences were observed between B6 and B6.IDO-/- mice in regards to susceptibility to MAIDS progression, there may be differences in viral load. For example, in a w.t. LP-BM5 infected B6 mouse, IDO may not suppress the pathogenic $\mathrm{CD} 4^{+} \mathrm{T}$ cells that are necessary for disease initiation and progression [51], but rather IDO may have an effect on viral spread and subsequent viral load. To address this question, splenic mRNA was extracted from uninfected and infected B6 and B6.IDO-/- mice. Splenic mRNA was assessed for LP-BM5-associated ecotropic (Eco) helper and defective (Def) Gag viral sequences. Compared to essentially no detectable signal in uninfected mice, a significant increase in Eco Gag and Def Gag mRNA was detected in B6 and B6.IDO-/- mice (Figure 4). Furthermore, no significant difference was observed between either Eco Gag or Def Gag of the infected B6.IDO-/- mice in comparison to infected B6 mice. Thus, we could not detect differences in accumulated retroviral load, suggesting that
LP-BM5 viral replication and spread were not substantially altered in B6.IDO-/- mice.

In summary, we demonstrated that B6.IDO-/- mice were susceptible to LP-BM5 induced disease and had relatively equivalent levels of disease as compared to infected B6 mice, at multiple timepoints and at varying doses. We further demonstrated that viral load was essentially unaltered in infected B6.IDO-/- mice in comparison to infected $\mathrm{B} 6$ mice. Our results are interesting to compare to those of Hoshi, et al., who recently reported an increase in IDO mRNA, protein levels, and enzymatic activity in response to LP-BM5 infection [31]. Their results are consistent with our results herein, as we demonstrated an increase in splenic IDO mRNA at 8 wpi (Figure 1A). However, it remains unclear whether viral infection per se and/or the host immune response is responsible for the induction of IDO. In any event, regardless of the source(s) of the induced IDO, this response does not appear, in itself, to be crucial in the balance of host resistance factors versus retroviral pathogenesis mechanisms that lead to the profound disease observed in LP-BM5 infected, susceptible B6 mice.

Hoshi, et al. also infected B6.IDO-/- mice or treated infected B6 mice with 1-methyl tryptophan (1MT), an IDO inhibitor, and demonstrated an increased responsiveness to the T-cell mitogen ConA, in comparison to infected B6 mice, suggesting decreased immunosuppression when IDO activity was not present [31]. However, no uninfected control mice were reported for comparison, thus making it unclear what percentage of mitogen responsiveness was restored in IDO deficient mice. In contrast, our data demonstrate that B6.IDO-/- mice

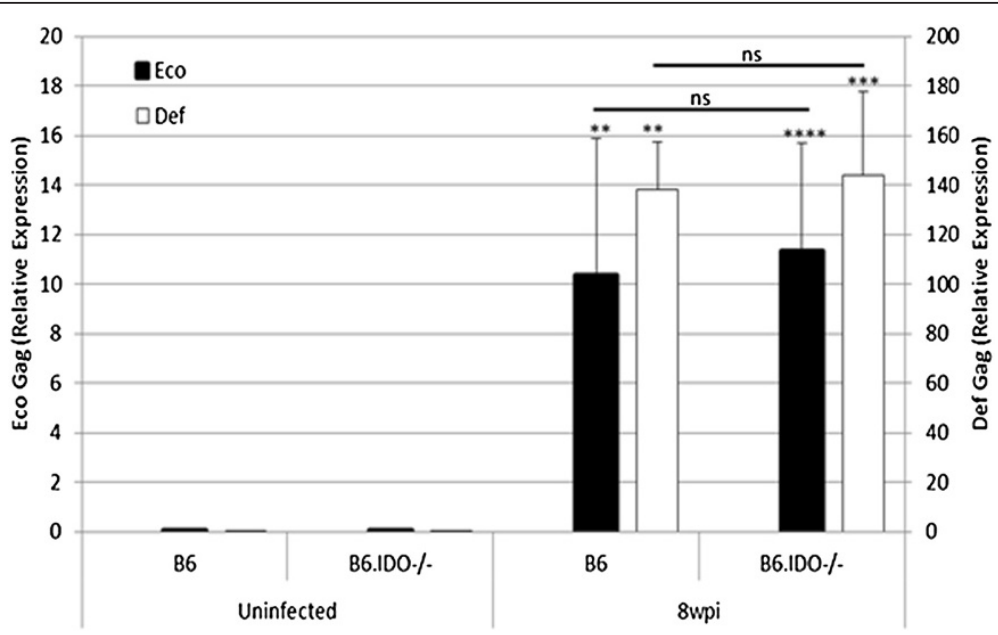

Figure 4 Infected B6.IDO-/- Mice Do Not Display An Altered LP-BM5 Viral Load. B6 and B6.IDO-/- mice were infected in parallel, with $5 \times 10^{4} \mathrm{pfu}$, and splenic mRNA was extracted at 8 wpi. LP-BM5 Eco Gag and Def Gag mRNA were assessed by qRT-PCR, relative to $\beta$-actin [63]. Histograms represent the means, $(n=4)$, with indicated standard deviations. The patterns of the presented results are representative of three additional experiments, which provided a similar pattern of results. ${ }^{* *} \mathrm{p}<0.01 ;{ }^{* *} \mathrm{p}<0.001$; ${ }^{* * *} \mathrm{p}<0.0001$, in comparison to uninfected controls, as determined using one-way ANOVA. 
exhibited substantially decreased responsiveness to both T-cell (ConA) and B-cell (LPS) mitogens in comparison to stimulated uninfected controls, and there was no difference in the extent of immunodeficiency as compared to infected w.t. mice (Figure 2E).

Due to these differential results in LP-BM5-induced immunosuppression between our data and those of Hoshi, et al., we employed a more comprehensive, yet accepted, panel of MAIDS parameters, to obtain a broader understanding of the role of IDO in LP-BM5-induced disease. All MAIDS parameters examined herein indicated LP-BM5-induced pathogenesis in infected B6.IDO-/- at roughly equivalent levels to those of infected B6 mice (Figures 2 and 3). Hoshi, et al. also reported decreased splenic viral copies of Def Gag at 8 wpi in either infected B6.IDO-/- mice or infected B6 mice treated with 1MT [31]. In our studies, we found no significant difference in splenic mRNA for Def Gag and Eco Gag at any of the tested timepoints (3, 5, and $8 \mathrm{wpi}$ ) (Figure 4 and data not shown). Although our LP-BM5 inoculum and that used by Hoshi, et al. appear to be comparable doses, we wanted to confirm that the effects seen were not due to our administration of a larger viral dose. To assess this, we compared three infectious viral doses, including two that were lower than our standard inoculum: $5 \times 10^{4}, 1 \times 10^{4}$, and $5 \times 10^{3}$ pfu. However, at each dose essentially no difference in splenic mRNA for Def or Eco Gag was observed between infected B6 and B6.IDO-/mice (data not shown), consistent with our finding of no differential levels of disease (Figure 3B). Alternatively, variance in the proportion of the defective and ecotropic genomes within the different LP-BM5 viral preparations might explain the differences seen between the two studies. Whether this potential variable of the pathogenic LP-BM5 Def Gag content or distribution among virions is responsible for the different results obtained here, versus by Hoshi, et al., or whether other factors are also responsible, is unclear. Future studies to clarify the differences may be informative for a better understanding of the regulation of LP-BM5-induced pathogenesis. In conclusion, our data clearly demonstrate a non-essential role of IDO in MAIDS or viral load development following LP-BM5 infection of susceptible B6 mice.

It is also instructive to consider the effects of IDO, or its absence, in other viral systems. IDO has been shown to have differential effects during HSV-1 infection. In vitro studies have demonstrated that IDO can exert an anti-viral effect against HSV-1 infection, which appears to be mediated in part by IFN $\gamma$ and/or IL-1 [52,53]. It was further demonstrated in vivo that mice infected with HSV-1, which consequently developed signs of encephalitis, had increased levels of quinolic acid, a downstream metabolite of tryptophan catabolism [54]. These studies suggest an anti-viral role for IDO during HSV-1 infection. However, treatment of mice with $1 \mathrm{MT}$ during HSV-1 infection had no detectable effect on HSV-1 replication or on the survival of the infected mice [55]. Thus, IDO may act as an immunosuppressive molecule during viral infections depending on the model of infection (in vitro versus in vivo), and, most likely, also by the presence/absence of other immunomodulatory signals within the micro-environment.

With respect to other immunodeficiency-causing retroviruses, IDO has been implicated as a potential therapeutic target against HIV/AIDS, and the use of IDO inhibitors has recently been explored within SIV models, but with mixed results. In vitro studies have shown improved proliferative responses of $\mathrm{CD}^{+}$and $\mathrm{CD}^{+} \mathrm{T}$-cells from immunosuppressed HIV-infected patients by addition of $1 \mathrm{MT}[27,33,56]$. There is also in vitro evidence that IDO acts differentially to suppress the T-cell response against HIV infection: IDO can arrest $\mathrm{CD} 4^{+} \mathrm{T}$ cells in the G1-S transition phase of the cell cycle, but can suppress $\mathrm{CD}^{+} \mathrm{T}$ cells by reduced expression of the CD28 costimulatory molecule [56]. In contrast, in vivo studies using $1 \mathrm{MT}$, to inhibit IDO, have led to variable results. In a murine model of HIV-induced encephalitis, in which human peripheral-blood lymphocytes (hu-PBLs) are given to nonobese diabetic-severe combined immunodeficient (NOD/SCID) mice and then are infected by injection of HIV-1-infected monocyte-derived macrophages, treatment with $1 \mathrm{MT}$ lead to an increase in the HIV-specific CTL response and essentially elimination of HIV-infected macrophages from the brain [33]. Similarly, SIV-infected rhesus macaques that were treated with $1 \mathrm{MT}$, and also on antiretroviral treatment (ART), displayed reduced SIV RNA levels in the spleen and plasma [57]. In this same study, however, macaques not on ART and treated with $1 \mathrm{MT}$ exhibited no change in viral levels [57]. Likewise, ART-treated macaques also given 1MT therapy demonstrated transiently increased plasma levels of tryptophan, but no reduction in kynurenine [57]. In a seemingly similar study, different results were obtained: SIV-infected ART-treated macaques also given 1MT demonstrated no effect on tryptophan catabolism or on viral load [58]. Clearly, more work still needs to be done to fully understand the potential use of $1 \mathrm{MT}$ or other agents that target IDO, or the pathways involving IDO, before its potential to treat HIV/AIDS patients can be determined. Therefore, the study of well-controlled animal models of retrovirusinduced immunodeficiency will undoubtedly be important to sort out these complex interactions and sometimes conflicting initial results.

\section{Conclusions}

Our data demonstrate a non-essential role of IDO in MAIDS or viral load following LP-BM5 infection of susceptible C57BL/6 mice. 


\section{Methods \\ Mice}

C57BL/6 (B6) mice were purchased from the National Cancer Institute (NCI, Bethesda, MD) and housed in the Center for Comparative Medicine and Research (CCMR) at the Geisel School of Medicine at Dartmouth. Breeding pairs of B6.IDO-/- mice were purchased from The Jackson Laboratory (Ban Harbor, ME) and bred in-house at the CCMR. All animal experiments were done with the approval of the Institutional Animal Care and Use Committee of Dartmouth College, and in conjunction with the Dartmouth Center for Comparative Medicine and Research, an AALAC approved animal facility. This institution has an Animal Welfare Assurance on file (A3259-01). The genetic backgrounds of B6.IDO-/mice were assessed at the DartMouse ${ }^{\mathrm{Tm}}$ Speed Congenic Core Facility at the Geisel School of Medicine at Dartmouth, which uses the Illumina, Inc. (San Diego, CA) GoldenGate Genotyping Assay to interrogate 1449 SNPs spread throughout the genome. The raw SNP data were analyzed using DartMouse's SNaP-Map ${ }^{\mathrm{Tm}}$ and Map-Synth ${ }^{\mathrm{TM}}$ software, allowing the determination for each mouse of the genetic background at each SNP location.

\section{LP-BM5 virus inoculation}

LP-BM5 retrovirus was prepared as previously described $[59,60]$. All mice were given intraperitoneal injections between 6 and 8 weeks of age with one of the following doses: $5 \times 10^{4} \mathrm{pfu}, 1 \times 10^{4} \mathrm{pfu}$, or $5 \times 10^{3} \mathrm{pfu}$ of LP-BM5.

\section{Genotyping PCR}

Tail snips were taken and digested with $25 \mathrm{mM} \mathrm{NaOH}$ and $0.2 \mathrm{mM}$ EDTA for 1 hour at $98^{\circ} \mathrm{C} .40 \mathrm{mM}$ Tris $\mathrm{HCl}$ (pH5.5) was added to samples and supernatant was isolated after centrifugation. Genomic DNA was amplified by PCR using primers for both w.t. IDO and knockout IDO from The Jackson Laboratory website: w.t. IDO Primer 1: 5'-TGG AGC TGC CCG ACG C-3', w.t. IDO Primer 2: 5'-TAC CTT CCG AGC CCA GAC AC-3', knockout IDO Primer 1: 5'-CTT GGG TGG AGA GGC TAT TC-3', knockout IDO Primer 2: 5'-AGG TGA GAT GAC AGG AGA TC-3'. PCR products were run on a $1.5 \%$ agarose gel and visualized.

\section{Splenocyte responses to mitogens}

Splenocytes were isolated as previously described and responses to Concanavalin A (ConA) and lipopolysaccharide (LPS) mitogens were measured [51]. Briefly, splenocytes were plated in triplicate in a flat-bottom 96-well plate and stimulated with a final concentration in the well of $0.75 \mathrm{ug} / \mathrm{ml}$ of ConA, $10 \mathrm{ug} / \mathrm{ml}$ of LPS or supplemented media alone. After 72 hours, cells were pulsed with $1 \mathrm{uCi}$ of $\left[{ }^{3} \mathrm{H}\right]$ thymidine (Dupont NEN,
Boston, MA) and harvested 6 hrs later for assessment of thymidine incorporation.

\section{ELISA determination of serum Ig}

Serum hyper IgG2a and IgM were assessed by an enzyme-linked immunosorbent assay (ELISA) as detailed previously [61,62]. Affinity-purified goat anti-mouse IgG2a or IgM antibodies were used to coat 96-well plates (Southern Biotechnology Associates, Birmingham, $\mathrm{AL})$ and the ELISA was developed using phosphate substrate (p4744, Sigma-Aldrich).

\section{Flow cytometry}

Surface staining of splenocytes was performed as previously described [51]. Cells were stained with FITC-, PE-, PerCP- and APC- conjugated fluorescent antibodies and the resulting fluorescence was measured on FACSCalibur or FACSCanto Instruments (BD Biosciences) to detect murine CD4 (RM4-5), Thy1.2 (53-2.1), CD11b (M1/70), and Gr-1 (RB6-8C5) (BioLegend and BD Biosciences). Appropriate amounts of FITC-, PE-, PerCP- and APC- conjugated Ig isotypes were used as controls. Data were analyzed using FlowJo software (Tree Star, Inc.).

\section{RNA isolation and real-time quantitative PCR}

Total RNA was isolated from the spleen, for quantitative RT-PCR using RNeasy columns, including DNAse-I treatment (Qiagen, Valencia, CA). DNAse-free RNA was reverse transcribed using the iScript cDNA synthesis kit (Qiagen, Valencia, CA). qRT-PCR was performed using SYBR Green PCR Core kit (Applied Biosystems, Foster City, CA) on an iCyler iQ instrument (Bio-Rad, Hercules, CA). Relative expression of Eco Gag, Def Gag, and IDO were evaluated in comparison to $\beta$-actin controls as previously described [63]. IDO primers were forward 5'-AGA CCA CCA CAT AGA TGA AG-3' and reverse 5'CCA CCA ATA GAG AGA CGA GGA-3' as previously described [5].

\section{Calculation of disease index}

The disease index was calculated as previously described [41]. Briefly, the percent disease was calculated for each mouse after measurement of each of the standard panel of MAIDS disease parameters (splenomegaly, serum levels of IgG2a and IgM, responsiveness to ConA and LPS, and percent of Thy $1.2^{+} \mathrm{CD} 4^{+} \mathrm{T}$-cells). Disease index values were designated based on the percentages of disease for each parameter. Mice with less than $1 \%$ disease were considered negative for disease and given a value of 0 , mice with $1-20 \%$ disease were assigned a value of 0.5 , mice with $21-40 \%$ disease were given a value of 1 , mice with $41-60 \%$ disease were assigned a value of 2 , mice with $61-80 \%$ disease were given a value of 3 , mice with 81-100\% disease were assigned a value of 4 , and mice with disease greater than $100 \%$ were given a value of 5 . 


\section{Abbreviations}

IDO: Indoleamine 2,3-dioxygenase; MAIDS: Murine AIDS; HIV: Human immunodeficiency virus; SIV: Simian immunodeficiency virus.

\section{Competing interests}

The authors declare that they have no competing interests.

\section{Authors' contributions}

MAO and WRG conceived of the study and contributed to the design of the study. MAO performed all experiments, analyzed the data, and wrote the manuscript. Both authors read and approved the final manuscript.

\section{Acknowledgements}

We thank David Leib, Edward Usherwood, Cynthia Stevens, Kathy Green, James Cook, Jessica Rastad, and Whitney Fu for many helpful discussions and technical assistance.

This work was supported by the National Institutes of Health RO1 grant (CA50157) to WRG and NIH T32 training grant (Al007363), which supported MAO. Flow cytometry was performed at the DartLab: Immunoassay and Flow Cytometry Shared Resource at the Geisel School of Medicine at Dartmouth, which was established by equipment grants from the Fannie E. Rippel Foundation, the NIH Shared Instrument Program, and the Geisel School of Medicine at Dartmouth, and which is supported in part by Core Grant (CA23108) from the National Cancer Institute to the Norris Cotton Cancer Center and NIH/NCRR NIGMS COBRE P20 (RR16437) and P30 (GM103415) grants: the Center of Biomedical Research Excellence in Molecular, Cellular, and Translation Immunological Research (WRG principal investigator)

Received: 3 December 2012 Accepted: 6 May 2013

Published: 17 May 2013

\section{References}

1. Baban B, Hansen AM, Chandler PR, Manlapat A, Bingaman A, Kahler DJ, et al A minor population of splenic dendritic cells expressing CD19 mediates IDO-dependent T cell suppression via type I IFN signaling following B7 ligation. Int Immunol 2005, 17:909-919.

2. Boasso A, Vaccari M, Hryniewicz A, Fuchs D, Nacsa J, Cecchinato V, et al: Regulatory T-cell markers, indoleamine 2,3-dioxygenase, and virus levels in spleen and gut during progressive simian immunodeficiency virus infection. J Virol 2007, 81:11593-11603.

3. Grohmann $U$, Bronte $V$ : Control of immune response by amino acid metabolism. Immunol Rev 2010, 236:243-264.

4. Mellor A: Indoleamine 2,3 dioxygenase and regulation of T cell immunity. Biochem Biophys Res Commun 2005, 338:20-24

5. Manlapat AK, Kahler DJ, Chandler PR, Munn DH, Mellor AL: Cellautonomous control of interferon type I expression by indoleamine 2,3-dioxygenase in regulatory CD19+ dendritic cells. Eur J Immunol 2007, 37:1064-1071.

6. Baban B, Chandler PR, Johnson BA 3rd, Huang L, Li M, Sharpe ML, et al: Physiologic control of IDO competence in splenic dendritic cells. J Immunol 2011, 187:2329-2335.

7. Mellor AL, Munn DH: IDO expression by dendritic cells: tolerance and tryptophan catabolism. Nat Rev Immunol 2004, 4:762-774.

8. Kahler DJ, Mellor AL: T cell regulatory plasmacytoid dendritic cells expressing indoleamine 2,3 dioxygenase. Handb Exp Pharmacol 2009, 188:165-196.

9. Mackenzie $C R$, Heseler $K$, Müller A, Däubener W: Role of indoleamine 2,3-dioxygenase in antimicrobial defence and immuno-regulation: tryptophan depletion versus production of toxic kynurenines. Curr Drug Metab 2007, 8:237-244

10. Puccetti P: On watching the watchers: IDO and type I/II IFN. Eur J Immunol 2007, 37:876-879.

11. Samelson-Jones BJ, Yeh S-R: Interactions between nitric oxide and indoleamine 2,3-dioxygenase. Biochemistry 2006, 45:8527-8538.

12. Soliman H, Mediavilla-Varela M, Antonia S: Indoleamine 2,3-dioxygenase: is it an immune suppressor? Cancer J 2010, 16:354-359.

13. Favre D, Mold J, Hunt PW, Kanwar B, Loke P, Seu L, et al: Tryptophan catabolism by indoleamine 2,3-dioxygenase 1 alters the balance of $\mathrm{TH} 17$ to regulatory T cells in HIV disease. Sci Transl Med 2010, 2:32ra36.
14. Mellor AL, Baban B, Chandler PR, Manlapat A, Kahler DJ, Munn DH: Cutting edge: $\mathrm{CpG}$ oligonucleotides induce splenic CD19+ dendritic cells to acquire potent indoleamine 2,3-dioxygenase-dependent $T$ cell regulatory functions via IFN Type 1 signaling. J Immunol 2005, 175:5601-5605.

15. Katz JB, Muller AJ, Prendergast GC: Indoleamine 2,3-dioxygenase in T-cell tolerance and tumoral immune escape. Immunol Rev 2008, 222:206-221.

16. Outinen TK, Mäkelä SM, Ala-Houhala IO, Huhtala HSA, Hurme M, Libraty DH, et al: High activity of indoleamine 2,3-dioxygenase is associated with renal insufficiency in Puumala hantavirus induced nephropathia epidemica. J Med Virol 2011, 83:731-737.

17. Tetsutani K, To H, Torii M, Hisaeda H, Himeno K: Malaria parasite induces tryptophan-related immune suppression in mice. Parasitology 2007, 134:923-930.

18. Makala LHC, Baban B, Lemos H, El-Awady AR, Chandler PR, Hou D-Y, et al: Leishmania major attenuates host immunity by stimulating local indoleamine 2,3-dioxygenase expression. J Infect Dis 2011, 203:715-725.

19. Carlin JM, Borden EC, Byrne Gl: Interferon-induced indoleamine 2,3-dioxygenase activity inhibits Chlamydia psittaci replication in human macrophages. J Interferon Res 1989, 9:329-337.

20. Pantoja LG, Miller RD, Ramirez JA, Molestina RE, Summersgill JT: Inhibition of Chlamydia pneumoniae replication in human aortic smooth muscle cells by gamma interferon-induced indoleamine 2, 3-dioxygenase activity. Infect Immun 2000, 68:6478-6481.

21. Schmidt SK, Siepmann S, Kuhlmann K, Meyer HE, Metzger S, Pudelko S, et al: Influence of tryptophan contained in 1-methyl-tryptophan on antimicrobial and immunoregulatory functions of indoleamine 2,3-dioxygenase. PLoS One 2012, 7:e44797.

22. Adams $O$, Besken $K$, Oberdörfer $C$, Mackenzie $C R$, Rüssing $D$, Däubener W: Inhibition of human herpes simplex virus type 2 by interferon gamma and tumor necrosis factor alpha is mediated by indoleamine 2,3-dioxygenase. Microbes Infect 2004, 6:806-812.

23. Bodaghi B, Goureau O, Zipeto D, Laurent L, Virelizier J-L, Michelson S: Role of IFN- $\gamma$ indoleamine 2,3-dioxygenase and inducible nitric oxide synthase in the replication of human cytomegalovirus in retinal pigment epithelial cells. J Immunol 1999, 162:957-964.

24. Terajima M, Leporati AM: Role of indoleamine 2,3-dioxygenase in antiviral activity of interferon-gamma against vaccinia virus. Viral Immunol 2005, 18:722-729.

25. Mao R, Zhang J, Jiang D, Cai D, Levy JM, Cuconati A, et al: Indoleamine 2,3-dioxygenase mediates the antiviral effect of gamma interferon against hepatitis B virus in human hepatocyte-derived cells. J Virol 2011, 85:1048-1057.

26. Schröcksnadel K, Wirleitner B, Winkler C, Fuchs D: Monitoring tryptophan metabolism in chronic immune activation. Clin Chim Acta 2006, 364:82-90.

27. Boasso A, Herbeuval J-P, Hardy AW, Anderson SA, Dolan MJ, Fuchs D, et al: HIV inhibits CD4+ T-cell proliferation by inducing indoleamine 2,3dioxygenase in plasmacytoid dendritic cells. Blood 2007, 109:3351-3359.

28. Boasso A, Royle CM, Doumazos S, Aquino VN, Biasin M, Piacentini L, et al: Overactivation of plasmacytoid dendritic cells inhibits antiviral T-cell responses: a model for HIV immunopathogenesis. Blood 2011, 118:5152-5162

29. Fu X, Lawson MA, Kelley KW, Dantzer R: HIV-1 Tat activates indoleamine 2,3 dioxygenase in murine organotypic hippocampal slice cultures in a p38 mitogen-activated protein kinase-dependent manner. J Neuroinflammation 2011, 8:88.

30. Bosinger SE, Li Q, Gordon SN, Klatt NR, Duan L, Xu L, et al: Global genomic analysis reveals rapid control of a robust innate response in SIV-infected sooty mangabeys. J Clin Invest 2009, 119:3556-3572.

31. Hoshi M, Saito K, Hara A, Taguchi A, Ohtaki H, Tanaka R, et al: The absence of IDO upregulates type I IFN production, resulting in suppression of viral replication in the retrovirus-infected mouse. J Immunol 2010, 185:3305-3312.

32. Fuchs D, Möller AA, Reibnegger $G$, Werner ER, Werner-Felmayer G, Dierich MP, et al: Increased endogenous interferon-gamma and neopterin correlate with increased degradation of tryptophan in human immunodeficiency virus type 1 infection. Immunol Lett 1991, 28:207-211.

33. Potula R, Poluektova L, Knipe B, Chrastil J, Heilman D, Dou H, et al: Inhibition of indoleamine 2,3-dioxygenase (IDO) enhances elimination of 
virus-infected macrophages in an animal model of HIV-1 encephalitis. Blood 2005, 106:2382-2390

34. Mosier DE, Yetter RA, Morse HC 3rd: Retroviral induction of acute lymphoproliferative disease and profound immunosuppression in adult C57BL/6 mice. J Exp Med 1985, 161:766-784.

35. Buller RM, Yetter RA, Fredrickson TN, Morse HC 3rd: Abrogation of resistance to severe mousepox in C57BL/6 mice infected with LP-BM5 murine leukemia viruses. J Virol 1987, 61:383-387.

36. Klinman DM, Morse HC 3rd: Characteristics of B cell proliferation and activation in murine AIDS. J Immunol 1989, 142:1144-1149.

37. Khan IA, Green WR, Kasper LH, Green KA, Schwartzman JD: Immune CD8(+) $T$ cells prevent reactivation of Toxoplasma gondii infection in the immunocompromised host. Infect Immun 1999, 67:5869-5876.

38. Mayrand SM, Schwarz DA, Green WR: An alternative translational reading frame encodes an immunodominant retroviral CTL determinant expressed by an immunodeficiency-causing retrovirus. J Immuno/ 1998, 160:39-50.

39. Mosier DE, Yetter RA, Morse HC 3rd: Functional T lymphocytes are required for a murine retrovirus-induced immunodeficiency disease (MAIDS). J Exp Med 1987, 165:1737-1742.

40. Ho O, Green WR: Cytolytic CD8+ T cells directed against a cryptic epitope derived from a retroviral alternative reading frame confer disease protection. J Immunol 2006, 176:2470-2475.

41. Rutkowski MR, Ho O, Green WR: Defining the mechanism(s) of protection by cytolytic CD8 T cells against a cryptic epitope derived from a retroviral alternative reading frame. Virology 2009, 390:228-238.

42. Carlson TL, Green KA, Green WR: Alternative translational reading frames as a novel source of epitopes for an expanded CD8 T-cell repertoire: use of a retroviral system to assess the translational requirements for CTL recognition and lysis. Viral Immunol 2010, 23:577-583.

43. Green KA, Okazaki T, Honjo T, Cook WJ, Green WR: The programmed death-1 and interleukin-10 pathways play a down modulatory role in LPBM5 retrovirus-induced murine immunodeficiency syndrome. J Virol 2008, 82:2456-2469.

44. Li W, Green WR: Immunotherapy of murine retrovirus-induced acquired immunodeficiency by CD4 T regulatory cell depletion and PD-1 blockade. J Virol 2011, 85:13342-13353.

45. Clark S, Duggan J, Chakraborty J: Tsl and LP-BM5: a comparison of two murine retrovirus models for HIV. Viral Immunol 2001, 14:95-109.

46. Green KA, Cook WJ, Green WR: Myeloid-derived suppressor cells in murine retrovirus-induced AIDS: inhibition of the T-cell and B-cell responsiveness that define the immunodeficiency. J Virol 2013, 87(4):2058-2071.

47. Alberati-Giani D, Malherbe P, Ricciardi-Castagnoli P, Köhler C, Denis-Donini S, Cesura AM: Differential regulation of indoleamine 2,3-dioxygenase expression by nitric oxide and inflammatory mediators in IFN-gamma -activated murine macrophages and microglial cells. J Immunol 1997, 159:419-426.

48. Sekkaï D, Guittet O, Lemaire G, Tenu JP, Lepoivre M: Inhibition of nitric oxide synthase expression and activity in macrophages by 3hydroxyanthranilic acid, a tryptophan metabolite. Arch Biochem Biophys 1997, 340:117-123.

49. Green KA, Cook WJ, Sharpe AH, Green WR: The CD154/CD40 interaction required for retrovirus-induced murine immunodeficiency syndrome is not mediated by upregulation of the CD80/CD86 costimulatory molecules. J Virol 2002, 76:13106-13110.

50. Knoetig SM, Torrey TA, Naghashfar Z, McCarty T, Morse HC 3rd: CD19 signaling pathways play a major role for murine AIDS induction and progression. J Immunol 2002, 169:5607-5614.

51. Li W, Green WR: The role of CD4 T cells in the pathogenesis of murine AIDS. J Virol 2006, 80:5777-5789.

52. Adams O, Besken K, Oberdörfer C, MacKenzie CR, Takikawa O, Däubener W: Role of indoleamine-2,3-dioxygenase in alpha/beta and gamma interferon-mediated antiviral effects against herpes simplex virus infections. J Virol 2004, 78:2632-2636.

53. Heseler K, Spekker K, Schmidt SK, MacKenzie CR, Däubener W: Antimicrobial and immunoregulatory effects mediated by human lung cells: role of IFN-gamma-induced tryptophan degradation. FEMS Immunol Med Microbiol 2008, 52:273-281.

54. Reinhard JF Jr, Flanagan EM: The neurotoxin quinolinic acid is increased in spinal cords of mice with herpes simplex virus encephalitis. Adv Exp Med Biol 1996, 398:241-246.
55. Divanovic S, Sawtell NM, Trompette A, Warning Jl, Dias A, Cooper AM, et al: Opposing biological functions of tryptophan catabolizing enzymes during intracellular infection. J Infect Dis 2012, 205:152-161.

56. Boasso A, Hardy AW, Anderson SA, Dolan MJ, Shearer GM: HIV-induced type I interferon and tryptophan catabolism drive T cell dysfunction despite phenotypic activation. PLoS One 2008, 3:e2961.

57. Boasso A, Vaccari M, Fuchs D, Hardy AW, Tsai W-P, Tryniszewska E, et al: Combined effect of antiretroviral therapy and blockade of IDO in SIV-infected rhesus macaques. I Immunol 2009, 182:4313-4320.

58. Dunham RM, Gordon SN, Vaccari M, Piatak M, Huang Y, Deeks SG, et al: Preclinical evaluation of HIV eradication strategies in the simian immunodeficiency virus-infected rhesus macaque: A pilot study testing inhibition of indoleamine 2,3-dioxygenase. AIDS Res Hum Retroviruses 2012, 29(2):207-214.

59. Klinken SP, Fredrickson TN, Hartley JW, Yetter RA, Morse HC 3rd: Evolution of $B$ cell lineage lymphomas in mice with a retrovirus-induced immunodeficiency syndrome, MAIDS. J Immunol 1988, 140:1123-1131.

60. Green KA, Noelle RJ, Green WR: Evidence for a continued requirement for CD40/CD40 ligand (CD154) interactions in the progression of LP-BM5 retrovirus-induced murine AIDS. Virology 1998, 241:260-268.

61. Green KA, Noelle RJ, Durell BG, Green WR: Characterization of the CD154positive and CD40-positive cellular subsets required for pathogenesis in retrovirus-induced murine immunodeficiency. J Virol 2001, 75:3581-3589.

62. Rutkowski MR, Stevens CA, Green WR: Impaired memory CD8 T cell responses against an immunodominant retroviral cryptic epitope. Virology 2011, 412:256-268.

63. Cook WJ, Green KA, Obar JJ, Green WR: Quantitative analysis of LP-BM5 murine leukemia retrovirus RNA using real-time RT-PCR. J Virol Methods 2003, 108:49-58.

doi:10.1186/1743-422X-10-154

Cite this article as: $\mathrm{O}^{\prime} \mathrm{Connor}$ and Green: The role of indoleamine 2,3dioxygenase in LP-BPM5 murine retroviral disease progression. Virology Journal 2013 10:154.

\section{Submit your next manuscript to BioMed Central and take full advantage of:}

- Convenient online submission

- Thorough peer review

- No space constraints or color figure charges

- Immediate publication on acceptance

- Inclusion in PubMed, CAS, Scopus and Google Scholar

- Research which is freely available for redistribution
C Biomed Central 